\section{Covid-19: Mass testing in Slovakia may have helped cut infections}

\section{Elisabeth Mahase}

Covid-19 infections fell in Slovakia after the rollout of rapid population-wide testing, but experts are not sure how much of the drop was a result of testing, as other restrictions were introduced at the same time. ${ }^{1}$

A preliminary analysis of three rapid antigen testing rounds reported that prevalence of detected covid infections decreased by $58 \%$ (95\% confidence interval $57 \%$ to $58 \%$ ) within one week in the 45 counties of Slovakia that were subject to two rounds of mass testing. This drop increased to $61 \%$ when adjusted for geographical clustering, attendance rates, and the epidemiological situation at the time of the first round.

The authors, from the London School of Hygiene \& Tropical Medicine, UK, said that this decrease could not be explained solely by the other restrictions and that it highlighted the impact of isolating people who tested positive and quarantining the members of their household.

However, while experts said the paper showed that mass testing "can contribute to a reduction in cases," they said that it was difficult to separate the effect of other restrictions from the impact of the mass testing. They added that polymerase chain reaction testing was not used to confirm results, meaning that it was not clear how accurate the testing was.

\section{Rounds of testing}

Slovakia deployed around 20 ooo medical staff and 40000 non-medical staff to run the programme, which started with a pilot from 23 to 25 October and was followed by a round of national mass testing on 31 October and 1 November. High prevalence counties were then targeted with a subsequent round on 7 and 8 November.

More than five million tests were completed, but rather than the rapid antigen tests being self-administered-as in the mass testing pilot in Liverpool, England-this scheme involved swabbing by trained medical staff. It used the SD Biosensor Standard $Q$ antigen test, the first rapid point-of-care covid-19 test approved for emergency use by the World Health Organization. ${ }^{2}$ In contrast, the Liverpool pilot had used the Innova SARS-CoV-2 antigen rapid qualitative test, which may miss as many as half of covid-19 cases when used by members of the public. ${ }^{3}$

Although Slovakia's testing was not mandatory, residents who did not attend were told to stay at home for 10 days or until the next round of mass testing. Those who participated received a medical certificate confirming their infection status, and a negative test certificate was required by employers in order to enter workplaces, while other venues carried out random checks.
Anyone who tested positive was asked to quarantine for 10 days, along with all members of the same household and their self-traced contacts.

During the month when mass testing was introduced Slovakia also implemented other infection control measures, such as closing schools for certain age groups and restricting indoor hospitality and leisure activities.

The analysis of the data, released as a preprint, showed that $84-87 \%$ of the eligible population participated in each round and that 50466 tests came back positive. The proportion of positive tests was $3.91 \%$ (range across counties $3.12 \%$ to $4.84 \%$ ) in the pilot, $1.01 \%$ (0.13\% to $3.22 \%$ ) in round 1 , and $0.62 \%$ (0.28\% 1.65\%) in round 2.

\section{Interventions and lockdown}

Speaking at a Science Media Centre press briefing on 7 December, the researchers said that it was not possible to fully remove the effect of the lockdown and emphasised that the intervention should be looked at as a whole package.

Sian Griffiths, emeritus professor at the Chinese University of Hong Kong and past president of the UK Faculty of Public Health, said, "Mass testing was given in combination with lockdown, but the authors did not know whether it made any contribution, because areas received both lockdown and mass testing.

"This is similar to the initial data from Liverpool, where all areas with lockdown decreased their number of infections, and this decrease appears to be similar in areas with and without mass testing. There are lots of things that can affect the rate of infections, so it's very hard to interpret these findings."

Alexander Edwards, associate professor in biomedical technology at the University of Reading, said, "It's great to see assessment of mass testing programs, but it's not straightforward to interpret this preprint in terms of suggesting mass testing will work everywhere, nor will it be straightforward to work out how similar programmes could be used in the UK.

"As is pointed out by the authors, there is a lot going on at the same time, so attributing a fall in cases to mass testing alone is hard. Nevertheless, it includes some indication that a mass testing programme can contribute to a reduction in cases.”

He added that a "cost-benefit assessment would be helpful to work out the relative cost of mass testing versus other testing protocols or different interventions." 
1 Pavelka M, van-Zandvoort K, Abbott S, et al. The effectiveness of population-wide, rapid antigen test based screening in reducing SARS-CoV-2 infection prevalence in Slovakia. medRxiv (preprint) 2020. doi: 10.1101/2020.12.02.20240648. https://www.medrxiv.org/con-

tent/10.1101/2020.12.02.20240648v1.full.pdf.

2 Mahase E. Covid-19: 120 million rapid tests pledged to low and middle income countries. BMJ 2020;371:m3857. doi: 10.1136/bmj.m3857 pmid: 33008800

3 Wise J. Covid-19: Safety of lateral flow tests questioned after they are found to miss half of cases. BMJ 2020;371:m4744. doi: 10.1136/bmj.m4744 pmid: 33277265

This article is made freely available for use in accordance with BMJ's website terms and conditions for the duration of the covid-19 pandemic or until otherwise determined by BMJ. You may use, download and print the article for any lawful, non-commercial purpose (including text and data mining) provided that all copyright notices and trade marks are retained. 